\title{
All-cause mortality among recipients of electroconvulsive therapy
}

\author{
Register-based cohort study \\ TRINE MUNK-OLSEN, THOMAS MUNK LAURSEN, POUL VIDEBECH, \\ PREBEN BO MORTENSEN and RABEN ROSENBERG
}

\author{
Background Studies investigating \\ mortality secondary to electroconvulsive \\ therapy (ECT) are few.
}

\begin{abstract}
Aims To assess the risk of mortality from natural and unnatural causes among ECT recipients compared with other psychiatric in-patients over a 25-year period.
\end{abstract}

Method Register-based cohort study of all in-patients admitted to a psychiatric hospital from 1976 to 2000. Cause-specific mortality was analysed using log - linear Poisson regression.

Results There were 783 deceased inpatients who had received ECT compared with 578I who had not. Patients who had received ECT had a lower overall mortality rate from natural causes $(\mathrm{RR}=0.82,95 \% \mathrm{Cl} 0.74-0.90)$ but a slightly higher suicide rate $(R R=1.20,95 \% \mathrm{Cl} 0.99$ I.47), especially within the first 7 days after the last ECT treatment $(\mathrm{RR}=4.82,95 \% \mathrm{Cl}$ 2.12-10.95).

\section{Conclusions Further investigation of} the effect of ECTon physical health and the observed increased suicide rate immediately following treatment are needed, although the last finding is likely to result from selection bias.

Declaration of interest None. Funding detailed in Acknowledgements.
Electroconvulsive therapy (ECT) is an established and effective modality (Geddes, 2003) which is most commonly used for the treatment of major depression (Fink, 2001; Abrams, 2002; Munk-Olsen et al, 2006). It is considered life-saving (Geddes, 2003) because of its rapid effect compared with pharmacological treatments. Clinical guidelines in Denmark (Andersson et al, 2002), USA (American Psychiatric Association, 2001) and UK (National Institute for Clinical Excellence, 2003) recommend its use as a first choice treatment for patients with potentially life-threatening conditions. Today, ECT patients are older, more medically ill and more often medicationresistant than 50 years ago (McCall, 2001), which suggests that they might have worse mental and physical health than patients who are not receiving this treatment. Electroconvulsive therapy is also used almost exclusively for in-patients for whom suicide rates are known to be high during admission and immediately after discharge. No study has taken this potential confounding into account, and long-term suicide rates after ECT treatment remain largely unexplored (Prudic \& Sackeim, 1999). The aim of this study was to assess the risk of mortality from natural and unnatural causes among ECT recipients compared with other psychiatric in-patients over a 25 -year period at a large regional psychiatric hospital in Aarhus, Denmark.

\section{METHOD}

Data were obtained from different Danish registers and were linked using a unique personal identification number (the CPR number). All live-born children and new residents in Denmark are assigned a CPR number, and all information is registered using this number in all national registers (Pedersen et al, 2006).
The study population consisted of all patients admitted at least once to the Psychiatric Hospital, Aarhus, University Hospital of Aarhus during the study period April 1976 to October 2000. The sample did not include out-patients who only rarely receive ECT. Admissions at other hospitals were not included owing to a lack of information about the use of ECT. In total, 783 ECT recipients and 5781 other psychiatric in-patients died during the study period. Follow-up started from the first day of the first admission after 1 April 1976 and ended on 1 October 2000 or at the date of death, whichever came first.

\section{Registers}

Information about ECT at the Psychiatric Hospital in Aarhus was obtained from a local register created from written charts detailing every episode of ECT given at the hospital between March 1976 and August 2000. Details covered the treatment itself (seizure duration and treatment choice, i.e. bilateral or unilateral) and treatment dates. This register was previously used to describe predictors of first-time ECT recipients (Munk-Olsen et al, 2006), and documents that $95 \%$ of all treatments were unilateral, which is in line with the study by Stromgren (1973).

There are no private psychiatric inpatient facilities in Denmark, so the Danish Psychiatric Central Register contains information about all admissions to Danish psychiatric hospitals and psychiatric facilities since 1969 (Munk-Jorgensen \& Mortensen, 1997). Diagnoses were entered as ICD-8 codes (World Health Organization, 1967) until the end of December 1993 and as ICD-10 codes (World Health Organization, 1992) from January 1994 onwards.

Information on causes and time of death was obtained from the National Register of Causes of Death (Juel \& Helweg-Larsen, 1999). If the time of death was the same day that the patient had been discharged, we categorised the patient as being admitted when analysing the variable 'days since discharge'.

\section{Diagnoses}

ICD-8 and ICD-10 diagnoses (primary diagnoses) were divided into six diagnostic groups: schizophrenia (ICD-8, 295 (minus 295.79); ICD-10, F20); schizoaffective disorders (ICD-8, 295.79, 269.89; ICD-10, F25); bipolar disorders (ICD-8, 296.19, 296.39; ICD-10, F30, 31); unipolar 
depressive disorders (ICD-8, 296 (minus 296.19, 296.39, 296.89), 298 (minus 298.39); ICD-10, F32-39); other nonaffective psychosis (ICD-8, 297, 298.39, 301.83; ICD-10, F21-29 (minus F25); and 'other disorders' (remaining diagnoses).

\section{Data analysis}

Data were analysed using the log-linear Poisson regression with SAS GENMOD version 8.02 for Windows, with personyears as an offset variable (Laird \& Olivier, 1981; Andersen et al, 1993). All variables except gender were treated as timedependent. All relative risks (RR) were adjusted for gender, diagnosis, calendar period (5-year intervals) and age (10-year intervals). Wald's test was used to calculate 95\% confidence intervals (CIs); CIs not including 1.00 indicated a statistically significant difference (at a $5 \%$ level) from the reference group.

\section{RESULTS}

A total of 783 ECT patients died during the study period of around 25 years: 593 $(76 \%)$ from natural causes and 190 (24\%) from unnatural causes. The relative risk of mortality compared with the other psychiatric in-patients was $0.86(95 \%$ CI 0.79-0.94). Among the 783 deceased ECT patients, 447 (57\%) had been diagnosed with unipolar affective disorders, 178 (23\%) with bipolar affective disorders, 55 (7\%) with schizoaffective disorders, 46 (6\%) with schizophrenia, $9(1 \%)$ with other non-affective psychosis and $48(6 \%)$ with 'other disorders'.

\section{Mortality from natural causes}

The mortality rate from natural causes was lower for ECT patients than for other psychiatric in-patients $(\mathrm{RR}=0.82,95 \% \mathrm{CI}$ 0.74-0.90, Table 1). Data were reanalysed adjusting for days since last ECT. The first 7 days after receiving the last ECT were characterised by an increased mortality risk $(\mathrm{RR}=2.11,95 \%$ CI $0.94-4.74)$, but results were based on only 6 patients and CIs overlapped with 1.00 (indicating a nonsignificant finding at the $5 \%$ level).

Owing to the decrease in mortality from natural causes among ECT-treated patients (Table 1), we performed additional analyses including specific causes of death (Table 2) and found a decreased mortality rate for respiratory diseases $(R R=0.73$,

Table I Relative risk of mortality from natural causes among ECT patients and other psychiatric in-patients

\begin{tabular}{|c|c|c|c|c|}
\hline & \multirow[t]{2}{*}{$n$} & \multirow[t]{2}{*}{ Person-years } & \multicolumn{2}{|c|}{ Relative risk $(95 \% \mathrm{Cl})$} \\
\hline & & & $\begin{array}{c}\text { First } \\
\text { adjustment' }\end{array}$ & $\begin{array}{c}\text { Final } \\
\text { adjustment }^{2}\end{array}$ \\
\hline ECT patients & 593 & 20923 & $0.82(0.74-0.90)$ & \\
\hline Other psychiatric in-patients & 5018 & 188018 & Reference & \\
\hline \multicolumn{5}{|l|}{ Discharge status } \\
\hline Currently admitted & 562 & 9513 & $1.35(1.23-1.49)$ & $1.34(1.22-1.48)$ \\
\hline Discharged within past 7 days & 60 & 884 & $3.01(2.33-3.88)$ & $2.98(2.3 \mathrm{I}-3.86)$ \\
\hline Discharged within past $8-30$ days & 168 & 3079 & $2.34(2.01-2.74)$ & $2.35(2.0 \mathrm{I}-2.74)$ \\
\hline Discharged more than 30 days ago & 4821 & 195465 & Reference & Reference \\
\hline \multicolumn{5}{|l|}{ ECT treatment } \\
\hline Within past 7 days & 6 & 63 & & $2.11(0.94-4.74)$ \\
\hline Within past I-4 weeks & 9 & 207 & & $0.76(0.39-1.47)$ \\
\hline More than 4 weeks ago & 578 & 20316 & & $0.84(0.76-0.92)$ \\
\hline No ECT treatment & 5018 & 188356 & & Reference \\
\hline
\end{tabular}

ECT, electroconvulsive therapy.

I. Adjusted for gender, age (10-year intervals), calendar period (5-year intervals), diagnosis and previous admissions. 2. Adjusted for gender, age (10-year intervals), calendar period (5-year intervals), diagnosis, previous admissions, and days since last ECT treatment.

Table 2 Cause-specific mortality for ECT-treated patients'

\begin{tabular}{lcc}
\hline Cause of death & $n$ & Relative risk $(95 \% \mathrm{Cl})$ \\
\hline Unnatural causes & & \\
All unnatural causes & 190 & $1.10(0.92-\mathrm{I} .30)$ \\
Suicide & 149 & $1.20(0.99-\mathrm{I} .47)$ \\
Accidents & $4 \mathrm{I}$ & $0.80(0.56-\mathrm{I} .14)$ \\
Natural causes & & \\
All natural causes & 593 & $0.82(0.74-0.90)$ \\
Cardiovascular diseases & 163 & $0.85(0.70-\mathrm{I} .03)$ \\
Malignant neoplasms & 124 & $\mathrm{I} .01(0.8 \mathrm{I}-\mathrm{I} .25)$ \\
Diseases of old age including stroke & 90 & $0.98(0.76-\mathrm{I} .26)$ \\
Respiratory diseases & 77 & $0.73(0.55-0.95)$ \\
Other causes & 139 & $0.67(0.56-0.82)$ \\
\hline
\end{tabular}

ECT, electroconvulsive therapy.

l. Based on 20923 person-years.

2. Adjusted for gender, age (10-year intervals), calendar period (5-year intervals), diagnosis, previous admissions with other psychiatric in-patients as reference group.

$95 \%$ CI $0.55-0.95)$ and other natural causes $\quad(\mathrm{RR}=0.67,95 \%$ CI $0.56-0.82)$. Mortality from cardiovascular diseases was also lower among ECT recipients than among other in-patients $(\mathrm{RR}=0.85,95 \%$ CI 0.70-1.03), but the difference was not statistically significant. For malignant neoplasm and diseases related to old age (including stroke), there was no difference in mortality rates between ECT-treated and other in-patients (Table 2).

\section{Mortality from unnatural causes}

Overall, there was a slightly increased but statistically insignificant mortality rate from unnatural causes among ECT patients $(\mathrm{RR}=1.10,95 \%$ CI $0.92-1.30$, Table 2). A total of 41 ECT-treated patients had died from accidents but this did not translate into an increased risk compared with other in-patients $(\mathrm{RR}=0.80,95 \%$ CI $0.56-1.14$, Table 2).

A total of 149 ECT patients died by suicide during the study period, $112(75 \%)$ of whom had unipolar or bipolar affective disorder. Patients treated with ECT had a marginally significant trend towards an increased risk of dying by suicide $(\mathrm{RR}=1.20$, 95\% CI 0.99-1.47) compared with other in-patients (Table 2). 
Table 3 Relative risk of suicide among ECT patients and other psychiatric in-patients.

\begin{tabular}{lrcc}
\hline & $n$ & Person-years & Relative risk $(95 \% \mathrm{Cl})^{\prime}$ \\
\hline Days since discharge & & & \\
$\quad$ Currently admitted & 126 & 9513 & $2.93(2.38-3.59)$ \\
Discharged within past 7 days & 39 & 884 & $9.49(6.80-13.24)$ \\
Discharged within past 8-30 days & 66 & 3079 & $4.77(3.67-6.19)$ \\
Discharged more than 30 days ago & 681 & 195465 & Reference \\
ECT treatment ${ }^{2}$ & & & \\
Within past 7 days & 6 & 63 & $4.82(2.12-10.95)$ \\
Within past I-4 weeks & 7 & 207 & $1.48(0.69-3.16)$ \\
More than 4 weeks ago & 136 & 20316 & $1.23(1.01-1.52)$ \\
No ECT treatment & 763 & 188356 & Reference \\
\hline
\end{tabular}

ECT, electroconvulsive therapy.

I. Adjusted for gender, age (10-year intervals), calendar period (5-year intervals), diagnosis and previous admissions.

2. At Psychiatric Hospital, University Hospital of Aarhus.

Admission status and time since discharge are important confounders in the analysis of suicide in patients with affective disorders (Hoyer et al, 2004). The suicide rate in general (i.e. irrespective of ECT treatment) was almost 10 times higher if the patient had been discharged within the past 7 days before the suicide $(\mathrm{RR}=9.49$, 95\% CI 6.80-13.24, Table 3). Six patients had died by suicide within the first week after treatment (Table 3), three with unipolar depressive disorders, two with schizophrenia and one in the group with 'other disorders'. Patients treated with ECT in the past week had a greatly increased risk of suicide compared with other patients $(\mathrm{RR}=4.82,95 \%$ CI 2.12-10.95). The risk declined towards that of patients not treated with ECT (but was marginally elevated) when the last session of ECT was more than 4 weeks previously $(\mathrm{RR}=1.23$, 95\% CI 1.01-1.52).

The increased suicide risk among ECT patients led us to examine the effect of the number of treatments. This varied from 3 to 17 among those who had died by suicide less than 7 days after discharge. Of the 6 ECT patients who died by suicide, 4 had received 10 or more treatments. The number of treatments received was not different from that of ECT patients who had died by suicide more than 1 week after discharge or ECT patients who had not died by suicide.

\section{DISCUSSION}

\section{Mortality from natural causes}

The overall mortality rate from natural causes was lower among ECT than other in-patients. Analysis of cause-specific mortality revealed that ECT patients had a lower mortality from respiratory diseases and the group of remaining disorders, and a marginally lower mortality from cardiovascular diseases than patients never treated with ECT. Other studies have found similar, reduced rates among ECT patients (Avery \& Winokur, 1976; Prudic \& Sackeim, 1999; Geddes, 2003), but these studies were based on fewer ECT patients and shorter study periods. The consistency of our findings with these studies strengthens our belief that the reduced mortality from natural causes is not an artefact.

The observed decreased risk of mortality from natural causes in ECT recipients could be a result of selection bias if patients with poor physical health are less likely to receive the treatment. However, in Denmark the only contraindications to ECT have been cerebral and other aneurysms. The Danish guidelines are in line with recommendations from the American Psychiatric Association that 'no absolute medical contraindications to ECT exist' (American Psychiatric Association, 2001). Furthermore, many medical conditions are contraindications for pharmacological treatments (Andersson et al, 2002), and we therefore do not believe that selection bias has influenced this result.

Patients treated with ECT are a selected group. First, ECT remains the first choice for treatment-resistant depression (Thase $\&$ Rush, 1995) and where a rapid, definitive response is required because of the severity of a psychiatric or medical condition (American Psychiatric Association, 2001). Patients treated with ECT have longer hospital stays than other patients (Munk-Olsen et al, 2006), which testifies to the severity of their illness. Second, ECT is used for treating patients with severe medical conditions for which antidepressants cannot be used. However, these aspects do not explain the decrease in risk of mortality from natural causes but suggest that ECT patients should have a higher mortality owing to these selection mechanisms.

The decreased mortality from natural causes might result from the rapid effect of ECT on depression, which may be accompanied by a more quickly established, better overall well-being compared with treatment with pharmacological agents (Philibert et al, 1995; Prudic \& Sackeim, 1999). The decrease in mortality among ECT patients might arise because these patients respond positively to this kind of therapy and are therefore more likely to receive ECT again upon recurrence of symptoms. The repeated use of this modality and its rapid efficacy could produce the overall reduction in mortality (Prudic \& Sackeim, 1999). Another potential explanation might be that ECT patients are more likely to be admitted, which makes them less likely to become seriously ill without staff noticing and therefore more likely to receive quicker treatment and better care.

A single factor is unlikely to be the sole explanation for the decrease in mortality from natural causes among ECT-treated patients, and further studies focusing on different causes of death are therefore needed. Register data cannot be used to study whether ECT might have a positive effect on blood pressure, level of stress or other factors. The observed decrease in mortality from natural causes is unlikely to be a result of bias, but the effect of ECT on psychiatric patients' physical health needs further exploration.

Mortality risk from natural causes was decreased for ECT patients compared with other psychiatric in-patients, but this does not mean that their mortality rates were decreased compared with people never admitted to a psychiatric hospital. Psychiatric patients are known to have increased mortality (from natural and unnatural causes) compared with the general population (Harris \& Barraclough, 1998; Hoyer et al, 2000; Osby et al, 2001).

\section{Mortality from unnatural causes}

Overall, ECT patients had only a marginally significant increased suicide rate compared with other psychiatric in-patients. The 
present study did not compare suicide or other mortality rates with those of the general population. However, our results are in line with other national studies of Danish patients (Hoyer et al, 2000, 2004) that have included some of the present regional sample. These results are in line with the general literature on suicide risk in people with affective disorders (Harris \& Barraclough, 1998).

Some studies have focused on ECT safety and have described mortality rates directly associated with ECT (Shiwach et al, 2001; Nuttall et al, 2004). Shiwach et al (2001) reported that 30 of 8148 patients who received ECT during a 5-year period in Texas died within 14 days of ECT. Eight of these patients had died by suicide. The authors concluded that suicide might indicate failure of ECT. However, the severity of the psychiatric illness and the time required for the treatment to have a positive effect should be considered when assessing ECT as a preventative measure for suicide (Shiwach et al, 2001).

Our results should be interpreted with caution given the methodological limitations (i.e. the lack of information on variables associated with suicide) (Sharma, 2001). Our data came from registers and unfortunately no information was available on specific indications for receiving ECT, pharmacological treatments and dose, discontinuation of medication prior to a course of ECT, past suicide attempts, patient well-being before and after the treatment and whether patients were on pass (temporary leave) during the admission.

A total of six ECT-treated patients died by suicide within 1 week of receiving treatment. These patients did not receive less treatment than the other ECT patients (mean 10.50 treatments per series $v .10 .22$ for all ECT patients, range 3-17). The elevated risk of suicide was not therefore a result of early treatment discontinuation. Psychiatric admission and discharge are associated with an increased risk of suicide (Hoyer et al, 2004), as found in this study. However, the elevated suicide rates in relation to time since last ECT treatment persisted even when admission status and time since discharge were taken into account. Hence our findings can not be ascribed to the well-established general association between suicide and psychiatric admission status.

Unipolar affective disorder is a strong predictor for receiving ECT (Munk-Olsen et al, 2006), and risk of suicide is particularly high among people with affective disorders (Mortensen et al, 2000). A total of $73(49 \%)$ of the ECT patients who died by suicide during the study period had unipolar affective disorders. Since ECT is often administered to patients thought to be suicidal the risk might have been even higher if this group had not received ECT. Our results do not contradict the assertion that ECT might be under-utilised for suicidal patients (Nemeroff et al, 2001).

Others studies have reported a positive effect of ECT on short-term suicide rates, but it should be borne in mind that suicide risk has been a primary indication for the use of ECT since its introduction (Prudic \& Sackeim, 1999), and therefore one would expect an elevated suicide rate among ECT patients. In the present study suicide risk was marginally increased among ECT recipients and was mainly, although not exclusively, confined to the first week after treatment. Therefore we could speculate that ECT returns the risk to the same (high) level of other patients with severe depression. However, another explanation is that almost a third of patients reported suicidal thoughts and acts prior to ECT in a study by Kellner et al (2005), and if patients are assigned ECT because they are at risk of suicide this will introduce bias. This bias would be expected to be stronger close to the time at which the treatment was administered. We believe that this bias (confounding by indication) is a likely explanation for the moderately increased suicide risk among ECT patients in our study and the more marked increase shortly after treatment.

Suicidal intent in patients with depression is rapidly relieved by ECT (Kellner $e t$ $a l, 2005)$ and we can not exclude the possibility that the risk during the first week after the last ECT session would have been higher if ECT had not been given to this particular subgroup of patients. This does not, however, exclude the possibility that elevated suicide rates immediately following ECT might be because ECT produces rapid improvement of depressive symptoms such as psychomotor retardation but does not eliminate suicidal impulses. This would indicate that patients should be assessed and monitored particularly carefully during this first period after relief of the depressive symptoms.

\section{Implications}

Compared with other psychiatric inpatients, ECT patients had an overall decreased mortality rate from natural causes but only a slightly increased suicide rate. A reduced mortality rate from natural causes among ECT patients has also been found in other studies, and there is a need for further investigation of the treatment and its potential effect on physical health. Although ECT patients are psychologically and physically severely ill, the decrease in mortality from natural causes implies that the treatment does not endanger but rather may have a positive effect on physical health.

The increased suicide rate among ECT patients shortly after treatment is probably a result of bias but our results also suggest that assessment and monitoring of suicide risk, and continuation treatment (e.g. antidepressants) continue to be important after the relief of depressive symptoms and ECT has been terminated.

\section{ACKNOWLEDGEMENTS}

We thank Dr L. S. Stromgren who was the originator of the ECT register and Dr S. Bjerum for his help with data collection. This study was supported by the Stanley Medical Research Institute, The Danish Research Council (grant no. 22-020333) and the Health Insurance Foundation (Sygekassernes Helsefond, grant no. II/212-95). Psychiatric epidemiological research at the National Centre for Register-Based Research is in part funded through a collaborative agreement with the Centre for Basic Psychiatric Research, Aarhus, Denmark.

\section{REFERENCES}

Abrams, R. (2002) Electroconvulsive Therapy. Oxford University Press.

American Psychiatric Association (2001) The Practice of Electroconvulsive Therapy. Recommendations for Treatment, Training, and Privileging. APA

Andersen, P. K., Borgen, Ø., Gill, R. D., et al (1993) Statistical Models Based on Counting Processes. SpringerVerlag.

Andersson, J. E., Bolwig, T. G., Jha, S. K., et al (2002) ECT behandling i Danmark. I-108. Dansk Psykiatrisk Selskab.

Avery, D. \& Winokur, G. (1976) Mortality in depressed patients treated with electroconvulsive therapy and antidepressants. Archives of General Psychiatry, 33, 1029-1037

Fink, M. (200I) Convulsive therapy: a review of the first 55 years. Journal of Affective Disorders, 63, I- 15.

Geddes, J. (2003) Efficacy and safety of electroconvulsive therapy in depressive disorders: a systematic review and meta-analysis. Lancet, 361 799-808.

Harris, E. C. \& Barraclough, B. (1998) Excess mortality of mental disorder. British Journal of Psychiatry, 173, II-53. 
Høyer, E. H., Mortensen, P. B. \& Olesen, A.V. (2000) Mortality and causes of death in a total national sample of patients with affective disorders admitted for the first time between 1973 and 1993. British Journal of Psychiatry, 176, 76-82.

Høyer, E. H., Olesen, A.V. \& Mortensen, P. B. (2004) Suicide risk in patients hospitalised because of an affective disorder: a follow-up study, 1973-1993. Journal of Affective Disorders, 78, 209-217.

Juel, K. \& Helweg-Larsen, K. (1999) The Danish registers of causes of death. Danish Medical Bulletin, 46 354-357.

Kellner, C. H., Fink, M., Knapp, R., et al (2005) Relief of expressed suicidal intent by ECT: a consortium for research in ECT study. American Journal of Psychiatry, 162, 977-982.

Laird, N. \& Olivier, D. (198I) Covariance analysis of censored survival data using log-linear analysis techniques. Journal of the American Statistical Association, 76, 231-240.

McCall, W. v. (200I) Electroconvulsive therapy in the era of modern psychopharmacology. International Journa of Neuropsychopharmacology, 4, 315-324.

Mortensen, P. B., Agerbo, E., Erikson, T., et al (2000) Psychiatric illness and risk factors for suicide in Denmark. Lancet, 355, 9-12.

Munk-Jorgensen, P. \& Mortensen, P. (1997) The Danish Psychiatric Central Register. Danish Medical Bulletin, 44, 82-84.

Munk-Olsen, T., Laursen, T. M., Videbech, P., et al (2006) Electroconvulsive therapy: predictors and trends in utilization from 1976 to 2000. Journal of ECT, 22, 127-132.

National Institute for Clinical Excellence (2003) Guidance on the Use of Electroconvulsive Therapy. NICE.

TRINE MUNK-OLSEN, MSc, THOMAS MUNK LAURSEN, PhD, National Centre for Register-Based Research, University of Aarhus, Denmark; POUL VIDEBECH, DrMedSc, Centre for Basic Psychiatric Research, Psychiatric Hospital, Risskov, Denmark; PREBEN BO MORTENSEN, DrMedSc, National Centre for Register-Based Research, University of Aarhus, Denmark; RABEN ROSENBERG, DrMedSc, Centre for Basic Psychiatric Research, Psychiatric Hospital, Risskov, Denmark

Correspondence: Trine Munk-Olsen, Centre for Register-Based Research, University of Aarhus, Taasingegade I, DK-8000 Aarhus C, Denmark.Email: tmo@ncrr.dk

(First received 23 May 2006, final revision 10 November 2006, accepted 4 December 2006)

Nemeroff, C. B., Compton, M. T. \& Berger, J. (200I)

The depressed suicidal patient. Assessment and treatment. Annals of the New York Academy of Science, 932, $1-23$

Nuttall, G. A., Bowersox, M. R., Douglass, S. B., et al (2004) Morbidity and mortality in the use of electroconvulsive therapy. Journal of ECT, 20, 237-24l.

Osby, U., Brandt, L., Correia, N., et al (200I) Excess mortality in bipolar and unipolar disorder in Sweden. Archives of General Psychiatry, 58, 844-850.

Pedersen, C. B., Gøtzsche, H, Møller, J. Ø., et al (2006) The Danish Civil Registration System. A cohort of 8 million persons. Danish Medical Bulletin, 53, 44I449.

Philibert, R. A., Richards, L., Lynch, C. F., et al (1995) Effect of ECT on mortality and clinical outcome in geriatric unipolar depression. Journal of Clinical Psychiatry, 56, 390-394.

Prudic, J. \& Sackeim, H. A. (1999) Electroconvulsive therapy and suicide risk. Journal of Clinical Psychiatry, $\mathbf{6 0}$ (suppl. 2), 104-110.
Sharma, V. (200I) The effect of electroconvulsive therapy on suicide risk in patients with mood disorders. Canadian Journal of Psychiatry, 46, 704-709.

Shiwach, R. S., Reid, W. H. \& Carmody, T. J. (200I) An analysis of reported deaths following electroconvulsive therapy in Texas, 1993-1998. Psychiatric Services, 52, 1095-1097.

Stromgren, L. S. (1973) Unilateral versus bilateral electroconvulsive therapy. Investigations into the therapeutic effect in endogenous depression. Acto Psychiatrica Scandinavica Supplementum, 240, 8-65.

Thase, M. E. \& Rush, A. J. (1995) Treatment-resistant depression. In Psychopharmacology: The Fourth Generation of Progress (eds F. E. Bloom \& D. J. Kupfer), pp. 1081-1098. Raven Press.

World Health Organization (1967) International Classification of Diseases: Manual of the International Statistical Classification of Diseases, Injuries and Causes of Death (ICD-8).WHO.

World Health Organization (1992) The ICD-10 Classification of Mental and Behavioural Disorders: Clinical Descriptions and Diagnostic Guidelines. WHO. 